ARTICLE

https://doi.org/10.1038/s41467-019-13217-y

\title{
Neurons in the Nucleus papilio contribute to the control of eye movements during REM sleep
}

\author{
C. Gutierrez Herrera (10 1,2,4 , F. Girard ${ }^{3,4 \star}$, A. Bilella (1) ${ }^{3}$, T.C. Gent ${ }^{1}$, D.M. Roccaro-Waldmeyer (1) ${ }^{3}$, \\ A. Adamantidis ${ }^{1,2} \&$ M.R. Celio ${ }^{3 \star}$
}

Rapid eye movements (REM) are characteristic of the eponymous phase of sleep, yet the underlying motor commands remain an enigma. Here, we identified a cluster of CalbindinD28K-expressing neurons in the Nucleus papilio (NPCalb), located in the dorsal paragigantocellular nucleus, which are active during REM sleep and project to the three contralateral eye-muscle nuclei. The firing of opto-tagged NPCalb neurons is augmented prior to the onset of eye movements during REM sleep. Optogenetic activation of NPCalb neurons triggers eye movements selectively during REM sleep, while their genetic ablation or optogenetic silencing suppresses them. None of these perturbations led to a change in the duration of REM sleep episodes. Our study provides the first evidence for a brainstem premotor command contributing to the control of eye movements selectively during REM sleep in the mammalian brain.

\footnotetext{
${ }^{1}$ Center for Experimental Neurology, Department of Neurology, Inselspital, University of Bern, Freiburgstrasse 3, CH-3010 Bern, Switzerland. ${ }^{2}$ Department of Biomedical Research (DBMR), University of Bern, Murtenstrasse 50, CH-3008 Bern, Switzerland. ${ }^{3}$ Anatomy and Program in Neuroscience, Faculty of Science and Medicine, University of Fribourg, Rte. Albert Gockel 1, CH-1700 Fribourg, Switzerland. ${ }^{4}$ These authors contributed equally: C. Gutierrez Herrera, F. Girard. *email: franck.girard@unifr.ch; marco.celio@unifr.ch
} 
leep is characterised by rapid transitions between REM (rapid eye movement) and non-REM states, the former being hallmarked by rapid eye movements (EMs), atony of the postural muscles, desynchronized electro-encephalographic (EEG) activity and vivid dreaming ${ }^{1,2}$. Information appertaining to the pathways that underlie these phenomena is largely fragmentary. Until now, interest has focused mainly on fathoming the intricacies of the neuronal networks that regulate the genesis, the maintenance and the termination of REM sleep. This research has revealed the crucial involvement of several brainstem areas, located prevalently in the pons ${ }^{3}$. During REM sleep, postural motoneurons are inhibited, and muscles are relaxed by the activation of a circuit originating from the sublaterodorsal (SLD)/ subcoeruleus (SubC) nucleus of the pons ${ }^{1,4}$ The motoneurons that subserve the external eye muscles are no exception to this rule; although tonically inhibited, they are phasically activated ${ }^{5,6}$, and provoke the rapid EMs that are characteristic of REM sleep. Although the eyes move in tandem during REM sleep ${ }^{7}$, the movements are often unconjugated ${ }^{5}$. Temporally, they coincide with the generation of the PGO waves ${ }^{6,8}$ - endogenous signals that in rats might correspond to $\mathrm{P}$-waves-which originate in the $\mathrm{SLD} / \mathrm{SubC}^{9}$. In terms of direction and velocity, EMs during REM sleep differ from the saccades that are generated during wakefulness ${ }^{5,10}$. The source of the phasic motor commands that underlie the EMs of REM sleep is unknown ${ }^{11,12}$.
In this study, we identify a conserved cluster of CalbindinD28k (Calb)-immunoreactive neurons in the medulla oblongata. On the basis of the results gleaned from in vivo neuroanatomical and optogenetic experiments, we conclude that these neurons contribute to the control of EMs during REM sleep.

\section{Results}

The Nucleus papilio: a conserved cluster of Calbindinexpressing neurons. We have previously identified in the rat brain a symmetric cluster of Calb-expressing neurons, lodged at the dorsomedial boundaries of the dorsal paragigantocellular nucleus (DPGi), distal to the abducens nucleus $(6 \mathrm{~N})$, lateral to the medial longitudinal fascicle (mlf), and below the Nucleus prepositus hypoglossi $(\mathrm{Pr})^{13}$. This butterfly-shaped cluster of Calbexpressing neurons, here referred to as $\mathrm{NP}^{\mathrm{Calb}}$ (Nucleus papilio), is phylogenetically conserved in rodents (Fig. 1a-c), cats (not shown), monkeys (Fig. 1d), and humans (Fig. 1e, f). It spans a distance of $\sim 0.6 \mathrm{~mm}$ (Bregma: -5.8 to $-6.4 \mathrm{~mm}^{14}$ ) in mice, $\sim 1.9 \mathrm{~mm}$ (Bregma: -10.8 to $-12.7 \mathrm{~mm}^{15}$ ) in rats and $\sim 5-8 \mathrm{~mm}$ in humans, in which it originates close to the abducens nucleus and extends caudally. Using stereological techniques, we have estimated the cell density in the NPCalb per hemisphere to be $388.1 \pm 47.9$ neurons in mice, $849.1 \pm 122.1$ neurons in rats and $1776.1 \pm 116.7$ neurons in humans.

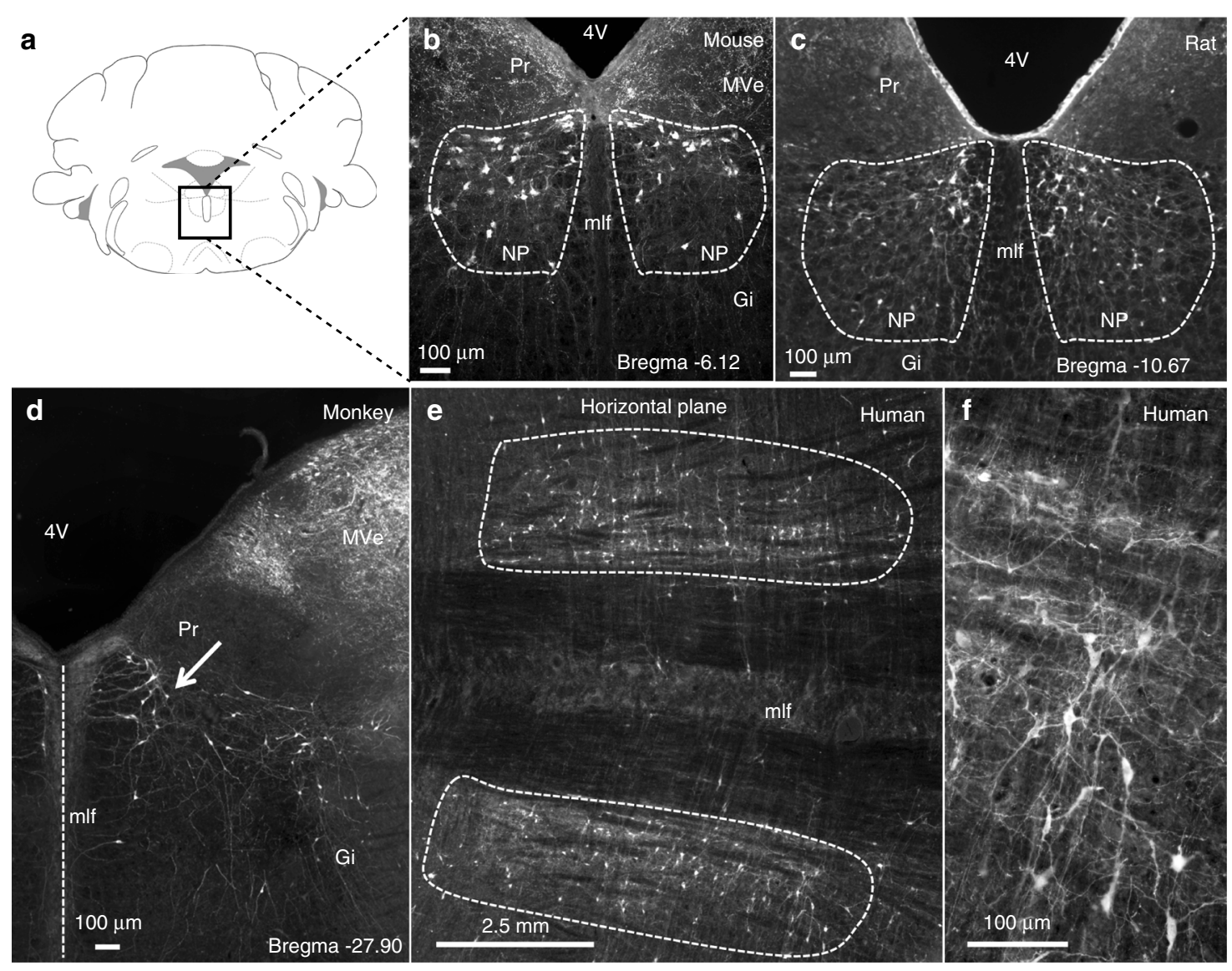

Fig. 1 The Nucleus papilio contains Calbindin-D28k expressing neurons. a Schematic representation of a coronal section through a rodent brain. b-f Calbimmunoreactivity is observed in neuronal cell bodies and processes in the NPCalb of mice $\mathbf{b}$, rat $\mathbf{c}$, monkeys $\mathbf{d}$ and humans $\mathbf{e}, \mathbf{f}$. The NPCalb is delineated with white dashed lines $\mathbf{b}, \mathbf{c}, \mathbf{e}$ or with an arrow $\mathbf{d}$. A vertical dashed line in $\mathbf{d}$ indicates the midline. The human brains $\mathbf{e}, \mathbf{f}$ were sectioned in the horizontal plane, the others in the coronal plane. $\mathbf{f}$ Higher magnification showing dendrites of Calb-immunoreactive neurons of the human NPCalb within the reticular formation. 4V: fourth ventricle; Pr: N. prepositus hypoglossi; mlf: medial longitudinal fasciculus; Gi: gigantocellular reticular nucleus; MVe: medial vestibular nucleus; NP: Nucleus papilio 
Activity of NPCalb neurons during REM sleep. The DPGi nucleus contains REM-on neurons (viz, those specifically activated during REM sleep) ${ }^{16}$, which play a role in the genesis of REM sleep via a GABAergic-mediated inhibition of wake-promoting neurons that are located in the Locus coeruleus (LC) and the dorsal raphe nucleus (DR ${ }^{17-20}$. Although chemical inactivation of these DPGi-neurons induces a prolonged period of wakefulness ${ }^{17}$, neurotoxic injury of the DPGi distal to the abducens region suppresses EMs without affecting the total duration of sleep or the bout duration of REM sleep ${ }^{21}$. After instigating a protocol of REM sleep deprivation and selective REM sleep rebound in both mice and rats, a large proportion of the NPCalb neurons were found to co-express c-fos, a marker of neuronal activity (the proportion of Calb-immunoreactive neurons expressing c-fos being $46.5 \pm 9.3 \%$ in rats and $42.2 \pm 5 \%$ in mice; Fig. $2 \mathrm{a}-\mathrm{c}$ ). Furthermore, $53.35 \pm$ $11.89 \%$ ( $n=4$ rats) of the $c$-fos-labelled neurons in the NPCalb failed to express Calb. These results were further supported by the single-cell electrophysiological recording of opto-tagged NPCalb neurons in freely moving mice. To achieve this end, we targeted the expression of channelrhodopsin-2 (ChR2) in NPCalb neurons by stereotactically injecting into the NPCalb of Calb1::Cre driver mice the adeno-associated virus encoding ChR2, which was fused to an enhanced yellow fluorescent protein reporter (AAV-EF1aDIO-hChR2(H134R)-YFP) (Fig. 2d). During REM sleep, the firing activity of the opto-tagged $\mathrm{NPCalb}$ neurons was augmented relative to that in other cells of the NPCalb area (viz., non-responders) (REM: $6.9 \pm 0.5 \mathrm{~Hz}$ in responder cells versus $0.025 \pm 0.0572 \mathrm{~Hz}$ in non-responders; Fig. 2e). Importantly, the firing rate of putative $\mathrm{NP}^{\mathrm{Calb}}$ neurons significantly increased prior to EMs during REM sleep, with a latency of $1.2 \pm 0.8 \mathrm{~s}(n=\sim 40$ EMs registered in 13 recording sessions in $n=4$ mice; Fig. $2 \mathrm{f}-\mathrm{h}$ ). Together, these data demonstrate that, during REM sleep, the activity of NPCalb neurons is time-locked to EMs.

Anterograde and retrograde tracing of $\mathrm{NP}^{\text {Calb }}$ neurons. To reveal the neuronal circuit that underlies the control of EMs during REM sleep, the efferences of NPCalb neurons were mapped by stereotactic injections of adeno-associated, Cre-dependent viral tracers (AAV2/1.CAG.FLEX.Tomato.WPRE.bGH or AAVEF1a-DIO-hChR2(H134R)-YFP) into the NPCalb of Calb1::Cre driver mice. Genetic targeting was specific for the Calbimmunoreactive neurons, stable and confined to the NPCalb neurons for at least 5 weeks after the injection (Fig. 3a top). Anterograde mapping (Table 1) revealed a large number of axonal endings in the contralateral nuclei that coordinate the EMs (oculomotor (III), trochlearis (IV) and, to a lesser degree, the abducens (VI)) (Fig. 3b; Supplementary Figs 1d and 2). Axonal terminals were also observed in the Darkschewitsch nucleus, the Roller nucleus (Supplementary Fig. 1b) and the raphe interpositus, the latter region composing the omnipause centre that blocks the EMs of the waking state ${ }^{22}$. The SLD/SubC-nuclei (involved in the generation of the rodent analogue of the PGO waves and in the induction of atonia in the postural muscles) and the pontine reticular nucleus $(\mathrm{PnC})$ (involved in the genesis and the maintenance of REM sleep) likewise receive inputs (Supplementary Fig. 1e, g). Terminals were also observed in the areas that are implicated in the processing of sensory inputs (nucleus of the trapezoid body, cuneate- and vestibular nuclei) and in lingual movements (hypoglossal nucleus) (Supplementary Fig. 1b, h). In contrast to the previously reported REM-on GABAergic neurons in the $\mathrm{DPGi}^{18}$, the $\mathrm{NPCalb}$ neurons project neither to the LC nor to the DR. The distribution in the eye-muscle nuclei of the axonal terminals from NPCalb neurons indicates a targeting of nerve cells supplying the single-innervated, twitch-muscle fibres ${ }^{23}$. We found that $\sim 33.8 \%$ of $\mathrm{NP}^{\mathrm{Calb}}$ neurons co-expressed the Slc17a6 gene transcripts (encoding the glutamate transporter VGlut2; Supplementary Fig. 3b). Their axonal terminals in the oculomotor nuclei manifested immunoreactivity for the VGlut2 protein (Supplementary Fig. 3c). No co-expression of the GABA transporter VGAT was observed (Supplementary Fig. 3b). Hence, this projection apparently utilises glutamate as a neurotransmitter. Furthermore, trans-synaptic retrograde mapping of NPCalb neurons using a Rabies virus (Env- $\Delta$ G-Rabies-GFP + AAV-B19G) revealed monosynaptic inputs from brain regions that are known to control REM sleep ${ }^{24,25}$, including the lateral hypothalamus (harbouring the REM sleep-promoting, melanin-concentratinghormone (MCH)-positive neurons), the SubC and the pontine reticular nuclei (PnC/PnO) (Table 2; Supplementary Fig. 4).

Involvement of NPCalb neurons in the control of EMs during REM sleep. Given the anatomical connections between the $\mathrm{NP}^{\mathrm{Calb}}$ neurons and the three ocular motor nuclei, the functional influence of these neurons on EMs was directly assessed using different but complementary experimental approaches.

Initially, we stereotactically injected the AAV-EF1a-DIOhChR2(H134R)-YFP virus into the NPCalb of Calb1::Cre mice. These mice were then chronically implanted with optical fibres, tetrodes and EEG/EMG/EOG electrodes for the simultaneous electrophysiological recording of single-unit activity across sleep-wake states in freely moving mice. The resulting expression of ChR2-eYFP was specific, and confined to the NPCalb neurons (Fig. 3a bottom) and their axonal terminals in the $3 \mathrm{~N}$ (Fig. 4b). Optical stimulation in freely moving mice reliably induced action potentials in ChR2-expressing NPCalb neurons during REM sleep and to a much lesser extent during wakefulness (Fig. 3c). Consistent with our correlative data, optogenetic activation of the ChR2-expressing NPCalb neurons upon $1 \mathrm{~s}$ continuous light, or $10 \mathrm{~Hz}$, stimuli consistently enhanced the probability of evoking EMs during REM sleep within a $1 \mathrm{~s}$ window relative to control conditions $(10 \mathrm{~Hz}$ : $58.6 \pm 5.5 \%$ vs $27.3 \pm 3.5 \%$; 1 s: $77.2 \pm 3.4 \%$ vs $17.2 \pm 3.5 \%$; Fig. $3 \mathrm{f}$ ); so too did optical stimulation with $1 \mathrm{~Hz}$ pulse, albeit to a lesser extent $(56.7 \pm 7.0 \%$ vs $32.9 \pm 3.0 \%$; Fig. 3 f). Average latencies between the optical stimulus and the onset of EMs were significantly shorter for the $1 \mathrm{~s}$-pulses than for the other stimuli in the ChR2-transduced mice relative to those in the control animals ( $1 \mathrm{~s}$ : $0.8 \pm 0.06 \mathrm{~s}$ vs $5.8 \pm 1.7 \mathrm{~s} ; 10 \mathrm{~Hz}: 1.8 \mathrm{~s} \pm 0.6 \mathrm{vs}$ $5.7 \pm 1.9 \mathrm{~s}, 1 \mathrm{~Hz}$ : $2.8 \pm 0.3 \mathrm{~s}$ vs $7.1 \pm 0.8 \mathrm{~s}$; Fig. $3 \mathrm{~g})$. When the optical stimuli were delivered during the state of wakefulness or during NREM sleep, no changes in the probability of, or latency to, EMs were observed in the ChR2-transduced mice relative to the control animals (Supplementary Fig. 5).

To ascertain whether a direct activation in the $3 \mathrm{~N}$ of the axonal terminals from NPCalb neurons support EMs during REM sleep, the NPCalb in Calb1::Cre mice was stereotactically injected with AAV-EF1a-DIO-hChR2(H134R)-YFP. The animals were chronically implanted with a single optical fibre, which was positioned just above the $3 \mathrm{~N}$ (Fig. 4a). Optogenetic activation of the CHR2expressing $\mathrm{NPCalb}$ neuronal terminals in the $3 \mathrm{~N}$ (Fig. 4b) significantly enhanced the probability of evoking EMs relative to control conditions during REM sleep $(76.17 \pm 3.6 \%$ vs $30.17 \pm$ $2.83 \%$ Fig. $4 \mathrm{e})$ but not during wakefulness $(44.94 \pm 4.66 \%$ vs $38.62 \pm 3.26 \%$ Fig. 4 e). These data are consistent with those gleaned from the optogenetic activation of the cell body. The latency to EMs was significantly shorter in this experiment (ChR2 vs Ctrl: $0.63 \pm 0.02 \mathrm{~s}$ vs $8.88 \pm 1.26 \mathrm{~s}$; Fig. $4 \mathrm{f}$ ), than in that targeting the activation of the cell body of NPCalb cells (ChR2 vs Ctrl: $1 \mathrm{~s}$ : $0.8 \pm 0.06 \mathrm{~s}$ vs $5.8 \pm 1.7 \mathrm{~s}$; Fig. $3 \mathrm{~g}$ ). Importantly, optogenetic activation of either the cell body or the axonal terminals of $\mathrm{NPCalb}$ neurons had no significant effect on either the duration (Fig. 4g, h, respectively) or the frequency of REM sleep episodes 
a

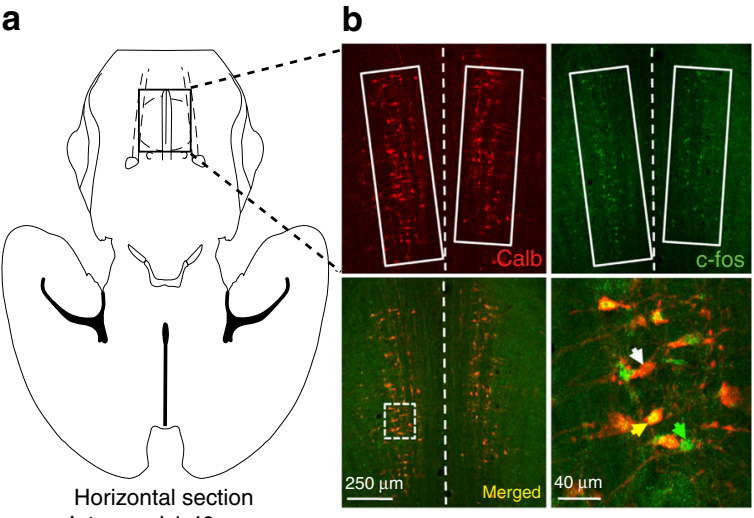
Interaural $1.40 \mathrm{~mm}$

d

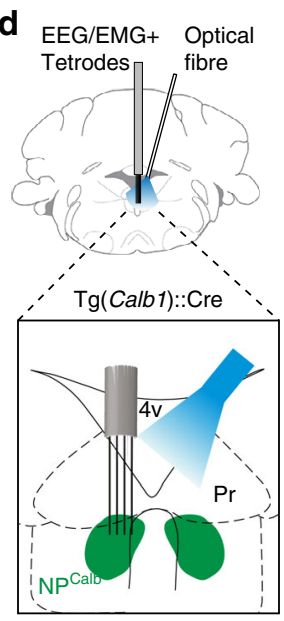

e

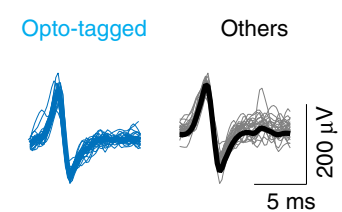

c

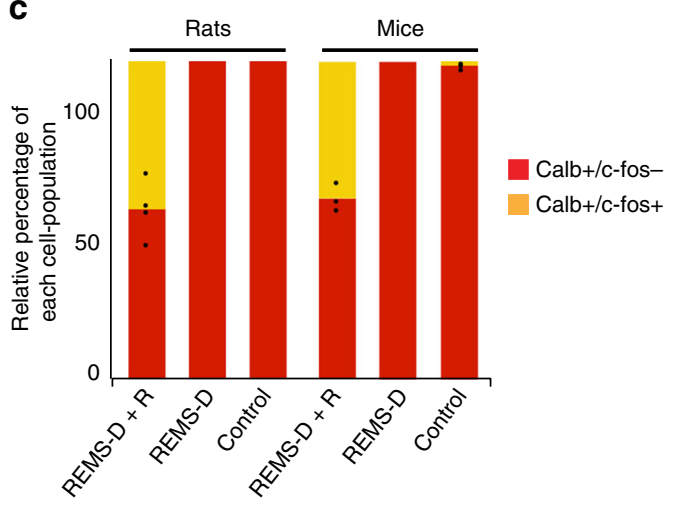

f
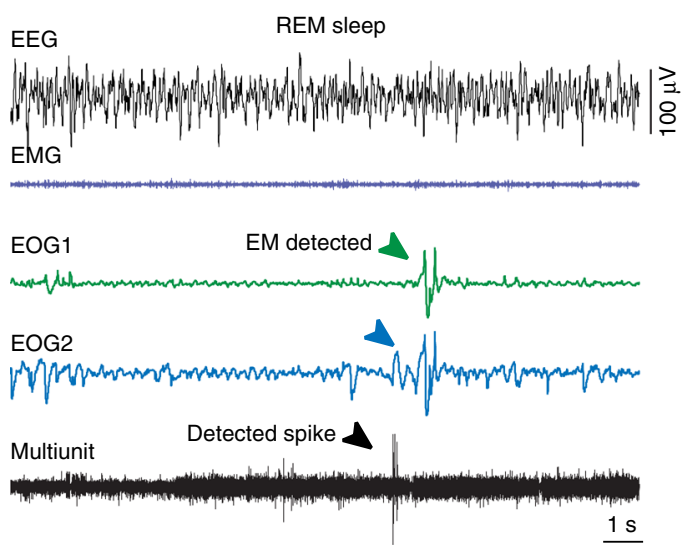

g

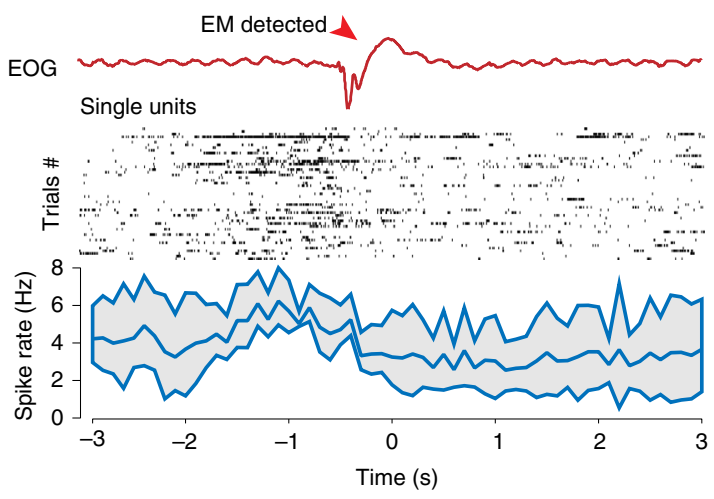

h

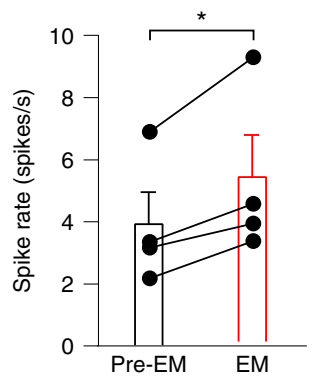

Fig. 2 Calbindin-D28k-expressing neurons of the Nucleus papilio are active during REM sleep. a Schematic representation of the localisation of the NPCalb in a rat horizontal brain section. b After a period of REM sleep deprivation, followed by a $3 \mathrm{~h}$ REM sleep rebound ("REMS-D+R"), a large subset of NPCalb neurons (boxed in white) expressed c-fos protein. Shown are representative examples of immunostaining in rat brain. A higher magnification view of the delineated area reveals the three populations of cells, namely, the neurons that are immunoreactive only for Calb (white arrow), those that are immunoreactive only for c-fos (green arrow), and those that are immunoreactive for both markers (yellow arrow). c Relative percentage of cell immunoreactive for $c$-fos in "REMS-D+R" ( $n=3$ mice and $n=4$ rats), "REMS-D" ( $n=3$ mice and $n=2$ rats) and "Control" ( $n=3$ mice and $n=3$ rats) groups. In the REMS-D+R group almost half of the Calb-immunoreactive neurons ( $42.2 \pm 5 \%$ in mice, and $46.5 \pm 9.3 \%$ in rats) were c-fos-positive. d Schematic representation of the movable tetrodes and the optical fibre implanted in the NPCalb. e NPCalb neurons were identified by their spiking response to single light pulses. Top: averaged neuronal spike rates of opto-tagged cells (blue) and non-responders (black) were similar. Bottom: averaged neuronal spike rates of opto-tagged cells (blue) and non-responders (black) across sleep-wake states. NPCalb neurons display significant lower firing rate than nonresponders during wake and the two populations have the opposite behaviour during REM sleep ( $n=4$ responder cells, and $n=6$ non-responder cells; four animals; wake: $P=0.0086, t=3.45$; NREM: $P=0.289, t=1.35$ and REM: $P \leq 0.0001, t=7.29$; $\mathrm{df}=8$; un-paired two-tailed $t$ test). $\mathbf{f}$ EEG/EMG representative trace during REM sleep, electro-oculogram (EOG) signals and detected EM (green/blue arrows) showing the correspondent multiunit activity and a representative detected spike (black arrow). $\mathbf{g}$ Top traces represent EOG, unfiltered signal. The raster plot of the spiking activity of opto-tagged NPCalb neurons (black) and the corresponding averaged spiking rates (blue trace) reveal the increase in firing activity that preceded the EMs during REM sleep. h Average summary data during REM sleep of the spiking rate of single-cell activity before and during EM (pre-EM = firing before EM: $3.901 \pm 1.033$ spikes/ $\mathrm{s} ; \mathrm{EM}=$ firing at $\mathrm{EM}: 5.299 \pm 1.353$ spikes $/ \mathrm{s} ; n=4$ cells; $P=0.0277 ; t=4.020 ; d f=3$ : two-tailed paired $t$ test). Error bars represent $\mathrm{SEM}$ values 
a

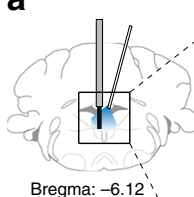
Bregma: -6.12
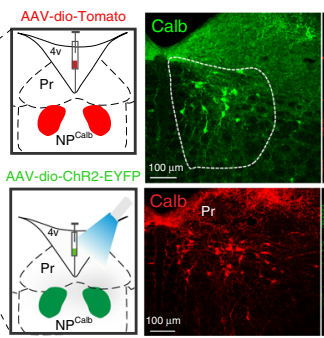
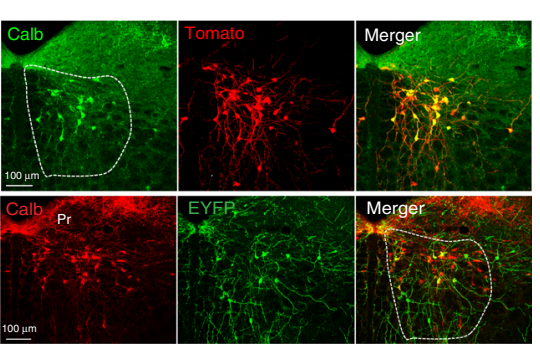

b

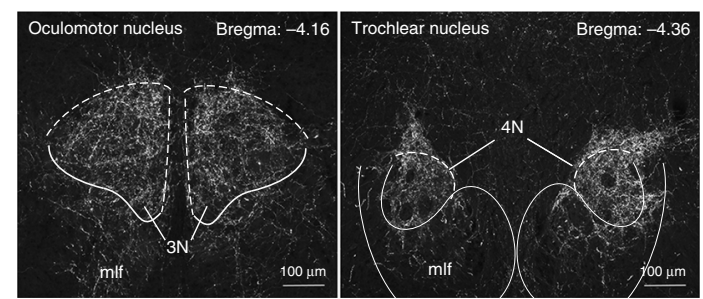

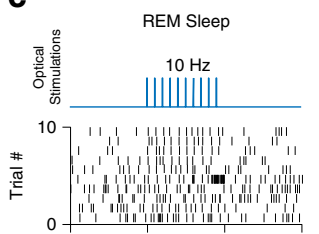

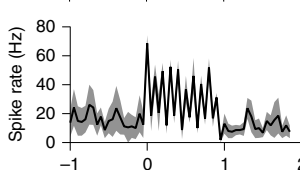

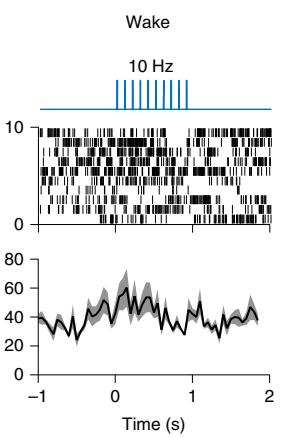

d

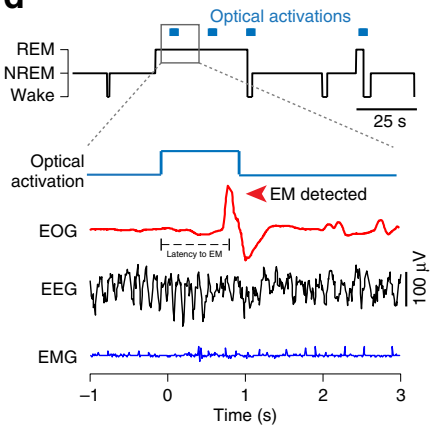

e

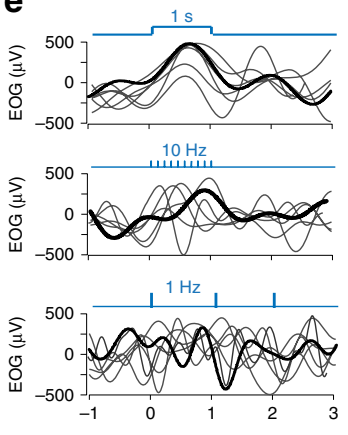

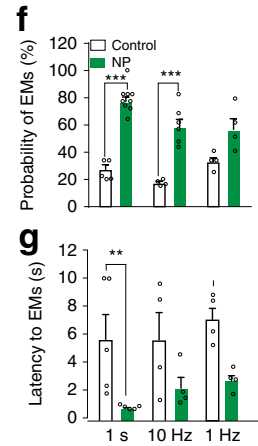

Fig. 3 Optogenetic activation of NPCalb neurons induces EMs during REM sleep. a Schematic representations of the stereotactic virus injections in the brain of Calb1::Cre mice (top: tracing experiment with Cre-dependent AAV-Tomato (AAV2/1.CAG.Flex.Tomato.WPRE.bGH); Bottom: optogenetics experiment with AAV-EF1a-DIO-hChR2(H134R)-YFP), tetrodes and optical fibre placement above the NPCalb (EEG/EMG/EOG electrodes are not shown). For each of the two experimental paradigms, representative images of coronal sections show expression of either Tomato and Calb (upper row) or ChR2-YFP and Calb (lower raw) in NPCalb neurons. The NPCalb region is delineated with white dashed lines. 4V: fourth ventricle; Pr: N. prepositus hypoglossi. b Dense Tomatoexpressing axon terminals of NPCalb neurons were found in the oculomotor ( $3 \mathrm{~N}$ ) and trochlear (4N) nuclei. The site of injection was median, resulting in the infection of NPCalb neurons in both hemispheres. mlf: medial longitudinal fasciculus. c Light-evoked spiking response of opto-tagged NPCalb cells during REM sleep (left) and wakefulness (right). Raster plots show the spiking activity of a representative NPCalb neuron during each state. Optical stimuli are shown on the top scheme. Bottom: averaged neuronal spike rates \pm SEM ( $n=4$ cells in four mice) during REM sleep and wakefulness. $\mathbf{d}$ Representative hypnogram of optogenetic activation of NPCalb neurons in vivo (top) together with EEG, EMG and EOG recordings. Red arrow indicates a light-evoked EM. The latency to EM is also indicated with a dashed line. e Overlay of averaged EOG recordings showing EMs triggered by optogenetic stimulation of NPCalb neurons at different frequencies during REM sleep. Grouped data and single animal values are shown in black and grey, respectively. $\mathbf{f}, \mathbf{g}$ Averaged probabilities $\mathbf{f}$ and latencies $\mathbf{g}$ of EMs in responses to optogenetic stimulations of NPCalb neurons during REM sleep. The data derived from at least five REM sleep episodes per stimulation frequency in ChR2-transduced and control animals (probability of EM: $1 \mathrm{~s}$ continuous light ( $n=9$ and 5 , respectively; $P=$ $<0.001, t=8.818, d f=26), 10 \mathrm{~Hz}(n=4$ and $6, P=<0.001, t=6.147, d f=26)$ and $1 \mathrm{~Hz}(n=4$ and $4 ; P=0.014, t=3.228, d f=26)$. Latency to EM: $1 \mathrm{~s}$ continuous light $(n=9$ and 5 , respectively; $P=0.036, t=3.765, d f=20), 10 \mathrm{~Hz}(n=4$ and $4 ; P=0.1103, t=2.219, d f=20)$ and $1 \mathrm{~Hz}(n=4$ and $4 ; P=$ $0.224, t=1.787, d f=20)) . P=0.12 \mathrm{~ns}\left({ }^{\star}\right), 0.33\left(^{\star}\right), 0.002\left({ }^{\star \star}\right),<0.001\left(^{\star \star \star}\right)$, two-way ANOVA followed by Sidak post hoc test for multiple comparisons. Error bars represent SEM values

(Supplementary Fig. 6), or on the parameters of either NREM sleep or wakefulness (Supplementary Fig. 6).

Finally, we adopted two different lack-of-function approaches to ascertain whether NPCalb neurons were necessary to generate the EMs during REM sleep. Initially, an adeno-associated virus encoding a Cre-dependent diphtheria toxin receptor (DTR) (AAV9-pCAG-Flex-DTR) was stereotactically delivered to the NPCalb of Calb1::Cre mice. The animals were then chronically implanted with EEG/EMG/EOG electrodes (Fig. 5a). After habituation to intraperitoneal (i.p.) injections of saline for 3 days, the diphtheria toxin (DTX) was administered i.p. on day 0, and EEG/EMG/EOG signals were continuously recorded for three successive days (Fig. 5b), after which period most of the NPCalb neurons were ablated ( $>93 \%$, Fig. $5 c$, d). During REM sleep, the number of EMs decreased markedly in the DTR-transduced mice relative to that in the control animals $(2.1 \pm 0.8$ vs $16.0 \pm 1.1$ on day 3; Fig. 5e, f), whereas they remained unchanged during wakefulness $(32.57 \pm 3.57$ vs $40.0 \pm 1.78$ on day 3 ; Fig. $5 \mathrm{~g})$. No statistically significant changes in sleep patterns were observed after DTX treatment in the DTR-transduced and the control animals (Supplementary Fig. 7). To substantiate our findings, the AAVdj-EF1alpha-DIO-ArchT3.0-EYFP virus encoding the archeorhodopsin 3.0 proton pump was stereotactically injected into the
NPCalb of Calb1::Cre mice to induce the transient optogenetic silencing of NPCalb neurons selectively during REM sleep in freely moving mice (Fig. 5h, i). Consistent with the genetic ablation experiment, optogenetic silencing of the NPCalb neurons induced a significant reduction in the total number of EMs that were evoked during a single episode of REM sleep in the ArchT3.0-transduced mice relative to that in the control animals ( $4.4 \pm 1.5$ vs $18 \pm 1.1$; Fig. 5k). The duration of an episode of REM sleep (Fig. 5l), and other sleep features (Supplementary Fig. 7), remained unaffected. Collectively, these results confirm the necessity of NPCalb neurons in the control of EMs, specifically during REM sleep.

\section{Discussion}

The data gleaned from investigations using different approaches, namely transectioning, lesioning or chemical inhibition, have established the pons to be the region of the brain responsible for the evocation of REM sleep, EMs, PGO-spikes and atonia ${ }^{1,8,26-31}$. For example, lesioning of the pedunculo-pontine tegmentum lead to a marked reduction in the number of EMs and of PGO-spikes ${ }^{30}$. However, some of these pontine lesions may have intersected the pons-traversing pathway from the $\mathrm{NPCalb}^{\mathrm{C}}$ in the upper medulla to the nuclei of the external eye muscles in the midbrain. 


\section{Table 1 Main efferent connections}

\section{Brain regions/identified nuclei-principal functions and pathways}

Medulla

$\mathrm{CeCv}$, central cervical nucleus

$M d D$, medullary reticular nucleus, dorsal

MdV, medullary reticular nucleus, ventral

$12 \mathrm{~N}$, hypoglossal nucleus-innervation of lingual muscles; regulated during sleep

Ro, nucleus of roller-part of circuits related to eye movements/sends imputs to III, IV and VI

LRt, lateral reticular nucleus

LPGi, lateral paragigantocellular nucleus-REM-on; proposed as inhibiting PS-off neurons of the LC; control of penile erection

$\mathrm{Gi}$, gigantocellular reticular nucleus-inhibition of motor neuron activity in the spinal cord during sleep

Spve/Mve/Lve/SuVe, spinal/medial/lateral/superior vestibular nucleus-coordination of head and eye movements

$\mathrm{MVePC} / \mathrm{MVeMC}$, medial vestibular nucleus parvicellular/magnocellular-coordination of head and eye movements

$6 \mathrm{~N}$, abducens nucleus (and Pa6, para-abducens nucleus)-oculomotor system (coordination of eye movements in the same direction)

Pons

Tz, nucleus of the trapezoid body-part of the auditory pathways

LSO, lateral superior olive-part of the auditory pathways

DPO, dorsal periolivary region-part of the auditory pathways

Bar, Barrington nucleus-principal micturition centre

RIP, raphe interpositus nucleus-premotor network for saccades (omnipause neurons)

SubC, subcoeruleus nucleus-induces muscle atonia during REM sleep; source of the PGO waves (rodents)

IRt, intermediate reticular nucleus

KF, Kölliker-Fuse nucleus-modulation of the respiratory function

$\mathrm{PnC}$, pontine reticular nucleus, caudal part-REM sleep initiation and maintenance; contains premotor neurons for saccades

Midbrain

$4 \mathrm{~N}$, trochlear nucleus (and $\mathrm{Pa} 4$, paratrochlear nucleus) - oculomotor system (innervate the superior oblique muscle of the contralateral eye)

$3 \mathrm{~N}$, oculomotor nucleus (and 3PC, oculomotor nucleus parvicellular) - oculomotor system (innervate external eye muscles except superior oblique and lateral rectus)

isRT/mRT, isthmic/mesencephalic reticular formation-involved in oculomotor function

Dk, nucleus of Darkschewitsch-one of the accessory oculomotor nuclei (eye movements, gaze coordination)

Summary of the main efferent connections of the Nucleus papilio identified by anterograde-tracing experiments with Cre-dependent AAV tracers in Calb1::Cre mice ( $n=5$ mice). The principal functions and pathways in which these nuclei are involved is indicated, with a special emphasis on sleep and eye movement regulation

\section{Table 2 Main afferent connections}

\section{Brain regions/identified nuclei}

\section{Medulla}

$\mathrm{MdD} / \mathrm{MdV}$, medullary reticular nucleus dorsal/ventral

$\mathrm{Sp} 5 \mathrm{C} / \mathrm{Sp} 5 \mathrm{I}$, spinal trigeminal nucleus caudal/interpolar

LPGi, lateral paragigantocellular reticular nucleus

$\mathrm{Gi}$, gigantocellular reticular nucleus

DPGi, dorsal paragigantocellular nucleus

Spve/Mve/Lve/SuVe, spinal/medial/lateral/superior vestibular nucleus

$\mathrm{MVePC} / \mathrm{MVeMC}$, medial vestibular nucleus parvicellular/magnocellular

Irt, intermediate reticular nucleus

Pons

SubC, subcoeruleus nucleus

$\mathrm{PnC}$, pontine reticular nucleus, caudal part

$\mathrm{PnO}$, pontine reticular nucleus, oral part

Midbrain

$\mathrm{mRT}$, mesencephalic reticular nucleus

Hypothalamus

rare sparse neurons in the hypothalamus

Summary of the main afferent connections to the Nucleus papilio identified by retrograde-tracing experiments with rabies virus in Calbi:::Cre mice $(n=5$ mice)

The DPGi, which was the focus of the present investigation, appears to be situated at the most caudal limit of this critical pontine region. The DGPi harbours two radically different types of neurons: the glutamatergic $\mathrm{NPCll}^{\mathrm{Calb}}$ neurons described here, and the GABAergic ones ${ }^{16-20}$. Although the latter are involved in the inhibition of waking neurons, particularly those from the LC, the $\mathrm{NPCalb}^{\mathrm{C}}$ neurons project excitatory connections to the nuclei of the external eye muscles.
The anatomical localisation of the $\mathrm{NPCalb}$ in the dorsomedial DPGi, together with its glutamatergic innervation of each of the three contralateral oculomotor nuclei that are responsible for the generation of EMs, distinguish these cells from the saccadegenerating premotor circuit that is responsible for the generation of EMs during wakefulness. The vertical and the torsional directions of the EMs during wakefulness are co-ordinated by the rostral interstitial nucleus of the medial longitudinal fascicle in the midbrain ${ }^{32}$, whereas those in the horizontal direction are under the control of excitatory, glutamatergic (EBNs) and inhibitory, glycinergic burst neurons (IBNs), which are respectively located rostral (EBNs) and just caudal (IBNs) to the $6 \mathrm{~N}^{33,34}$. Unlike the NPCalb, which is comprised of Calb/VGlut2-expressing neurons that do not co-express parvalbumin (Supplementary Fig. 3d), the EBNs and the IBNs do express this calcium-binding protein and are mantled with perineuronal nets ${ }^{22,35}$. Collectively, these differences afford evidence that EMs during REM sleep and wakefulness are, at least partly, controlled by distinct neuronal circuits. Nevertheless, the EOG is a rough readout of EMs, and our finding that the genetic ablation of NPCalb neurons does not significantly decrease the number of EMs that are evoked during wakefulness does not exclude the possibility that a more refined technique for measuring EMs might reveal subtle differences.

We report here that the NPCalb is conserved in rodents and primates, including humans, with respect both to its Calbimmunoreactivity and its anatomical position. Several imaging studies in humans (fMRI, tomography, PET) have disclosed the EMs that are evoked during the phases of wakefulness and REM sleep to share several neural correlates, including neuronal activation in the pons and the cortical frontal eye fields ${ }^{36,37}$. Neuronal activity in the medulla oblongata of humans has not hitherto been reported in association with EMs during REM sleep, perhaps owing to the technical difficulties of imaging this region of the brainstem. 
a

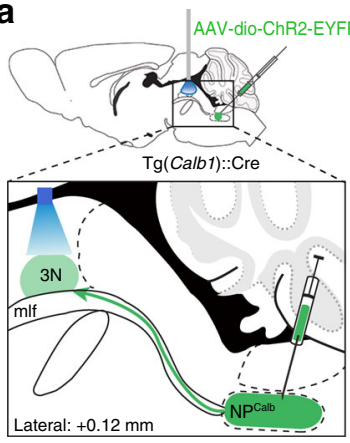

b

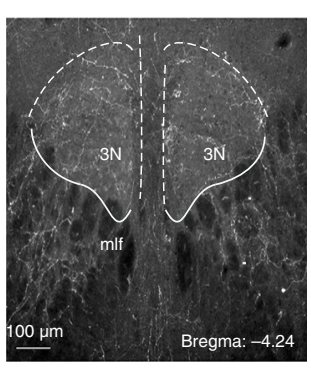

C
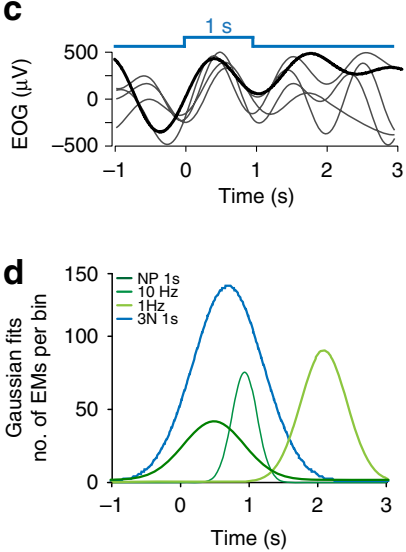

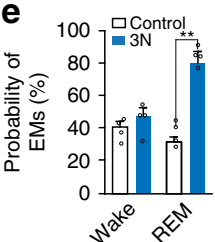

f

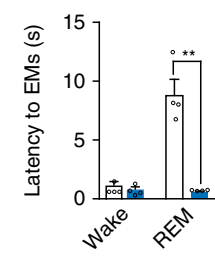

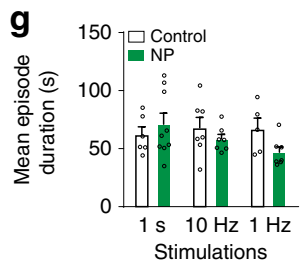

$\mathbf{h}$

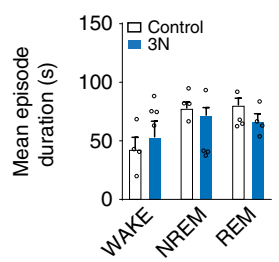

Fig. 4 Optogenetic activation of NPCalb terminals in the oculomotor nucleus. a Schematic representation of the experimental set up used to activate the axon terminals of NPCalb neurons in the oculomotor nucleus $(3 \mathrm{~N})$. The boxed area represents a higher magnification view of the injection of AAV-EF1aDIO-hChR2(H134R)-YFP in the NPCalb and the location of the optical fibre used for light delivery above the 3N. b Representative image of ChR2-EYFPpositive terminals in the $3 \mathrm{~N}$. c Overlay of averaged EOG recordings showing EMs triggered by optogenetic stimulation of NPCalb neuron terminals in the $3 \mathrm{~N}$ upon $1 \mathrm{~s}$ continuous illumination during REM sleep. Grouped data and single animal values are shown in black and grey, respectively. d Comparative Gaussian distribution of EOG signals triggered by optogenetic activation of NPCalb neuron soma $(n=6)$ or $3 \mathrm{~N}$ terminals $(n=4)$ per condition. Gaussian distribution is a fit to maximal deflection of EOG signals. e, $\mathbf{f}$ Averaged probabilities $\mathbf{e}$ and latencies $\mathbf{f}$ of EMs in response to optogenetic stimulations of NPCalb neuron terminals in $3 \mathrm{~N}$ during wakefulness and REM sleep $(n=4$ mice per group; $P=0.43, t=0.8895$ for wake; $P=0.0016, t=11.098$ for REM; $d f=3$ in both experimental conditions tested). ${ }^{\star} P<0.001$ significance was calculated using a paired $t$ test. $\mathbf{g}$ Summary of the mean duration of wake, NREM and REM sleep bouts, during a $1 \mathrm{~h}$ period of light activation delivered every minute of NPCalb cell soma at $1 \mathrm{~s}$ continous light $(n=9$ and $6, P=0.79, t=$ $0.832), 10 \mathrm{~Hz}(n=7$ and $7, P=0.69, t=0.876)$, and $1 \mathrm{~Hz}(n=7$ and $5, P=0.28, t=0.0 .65) ; d f=35$, when comparing ChR2-transduced to control animals. $\mathbf{h}$ Summary of the mean duration of wake, NREM and REM sleep bouts during a $1 \mathrm{~h}$ period of $1 \mathrm{~s}$ continuous stimulation delivered every minute to activate the NPCalb neuron terminals in $3 \mathrm{~N}$ ( $n=4$ in each group; $P=0.78, t=0.8691$ for wake; $P=0.941, t=0.517$ for NREM; and $P=0.592, t=1.167$ for REM; $d f=18$ when comparing ChR2-transduced to control animals). Neither the activation of the NPCalb cell soma nor the activation of their terminals in the $3 \mathrm{~N}$ had a significant effect on the duration of wakefulness, NREM and REM sleep episodes. Error bars represent SEM values

The findings of our optogenetic experiments reveal the firing rate of $\mathrm{NPCalb}$ neurons to increase prior to the generation of EMs, thus enhancing the probability of their evocation during REM sleep. The occurrence of a delay between the optogenetic activation of the NPCalb neurons and the manifestation of EMs is reminiscent of a similar delay that is observed between the stimulation of the SubC and the generation of PGO waves, which coincides temporally with the manifestation of $\mathrm{EMs}^{38}$.

Although no significant effects on the onset and the maintenance of REM sleep parameters were observed either upon the activation, the inhibition or the deletion of NPCalb neurons, further studies are required to ascertain whether the competence of these neurons-or of others intermingling Calb-negative ones in this region-transcend in scope its premotoric role in the generation of EMs during REM sleep.

In summary, our data furnish experimental evidence in a mouse model that NPCalb neurons and their projections to the oculomotor nuclei contribute to the control of EMs during REM sleep. The capability to induce EMs during REM sleep on command affords a powerful tool for the investigation of their functions.

\section{Methods}

Animals. For the morphological and the immunofluorescence analysis of the $\mathrm{NPCalb}$ in rodents, $8-12$ weeks old C57BL/6 J mice (from our animal facility) of both genders $(n=8)$ were utilised, as well 3 months old Wistar rats (Janvier, Lyon, France) ( $n=3$ males and three females).

The REM sleep deprivation experiments were conducted using nine female Wistar rats (Janvier, Lyon, France), and nine C57BL/6 J mice.

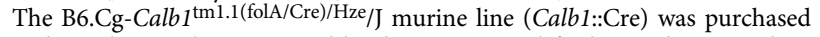
from the Jackson Laboratory, and bred in our animal facility under normal conditions. Genotyping was performed by PCR following the protocol of the supplier. For the anterograde-tracing experiments, five Calb1::Cre male mice were used. The injections were deemed to have been successful if the tracer was confined to the NPCalb neurons. For the retrograde-tracing experiments, five Calb1::Cre mice were employed. For analysing connections of the hypothalamic $\mathrm{MCH}$ neurons to the NPCalb, two Mch::Cre mice (Tg(Pmch-Cre) 1 Lowl, Jackson Laboratories, Bar Harbour, USA) were employed.

For studying the coexistence of Calb-expression with various markers for neurotransmitters, four mice each of three mouse lines obtained from Jackson

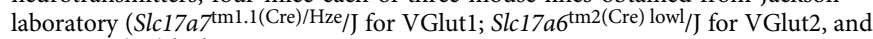

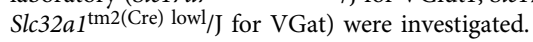

The optogenetic stimulation,-or inhibition-experiments were conducted with 13 Calb1::Cre male mice, and 9 control wild-type littermates. The full analysis was retrospectively limited to mice in which the injection of the AAV-ChR2 [AAV2EF1a-DIO-hChR2(H134R)-YFP] or AAV-ArchT [AAV2-Ef1a-DIO-ArchT3.0$\mathrm{EYFP}]$ viruses and its expression were limited to the $\mathrm{NPCalb}$.

The experiments consisting in eliminating NPCalb neurons by means of diphtheria toxin (AAV9-pCAG-Flex-DTR) injection were conducted on seven Calb1::Cre mice and four control littermates, injected in the NPCalb; the full analysis was limited to mice in which the injection of the virus was most precise

For in vivo recording of $\mathrm{NP}^{\mathrm{Calb}}$ neuron activity, four Calb1::Cre mice were used and for the optogenetic activation of the axonal endings of NPCalb neurons in the oculomotor nucleus, four Calb1::Cre male mice were employed.

The animals were housed in state-of-the-art animal facilities and in accordance with the relevant Swiss laws. The study was approved by the Committee for Animal Experimentation of the Cantons of Fribourg (2015-FR18), respectively, Bern (2015FR18+) (Switzerland).

For anatomical studies, the animals were anaesthetised with pentobarbital (100 $\mathrm{mg} / \mathrm{kg}$ of body weight). In a state of narcosis they were perfused via the left ventricle, first with chilled $\left(4^{\circ} \mathrm{C}\right)$ physiological $(0.9 \%)$ saline and then with chilled $\left(4^{\circ} \mathrm{C}\right) 4 \%$ paraformaldehyde. The brains were excised and post-fixed overnight in the same fixative. They were then immersed in $0.1 \mathrm{~m}$ Tris-buffer ( $\mathrm{pH} 7.3$ ) containing $20 \%$ sucrose in preparation for cryo-sectioning.

Specimens of the brains of Macaca mulatta and Macaca fascicularis, which had been killed for other purposes, were provided by Dr. Grazyna Wieczorek (Novartis, Basel, Switzerland)

Samples of human brains were supplied by Professor Luis Filgueira (Anatomy Unit, University of Fribourg, Switzerland). Specimen 2-15 and 7-15 were derived from male subjects, 70 and 80 years of age who had donated their body to anatomy for teaching and research purposes. They had manifested no signs of neurological disorders.

Immunofluorescence. Depending on the nature of the experiment, the various brain specimens were cryosectioned into $30-, 40-$, or 80 - $\mu \mathrm{m}$-thick sections (coronal or horizontal plane), which were collected directly in $0.1 \mathrm{~m}$ Tris-buffer containing 
a

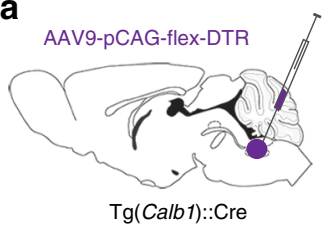

b

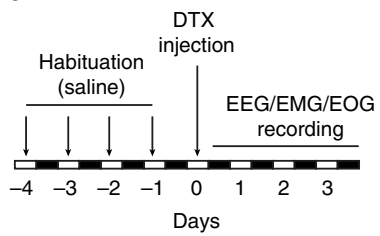

C

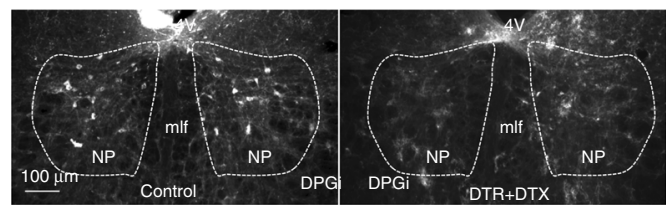

d

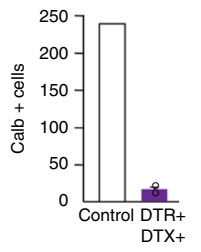

e

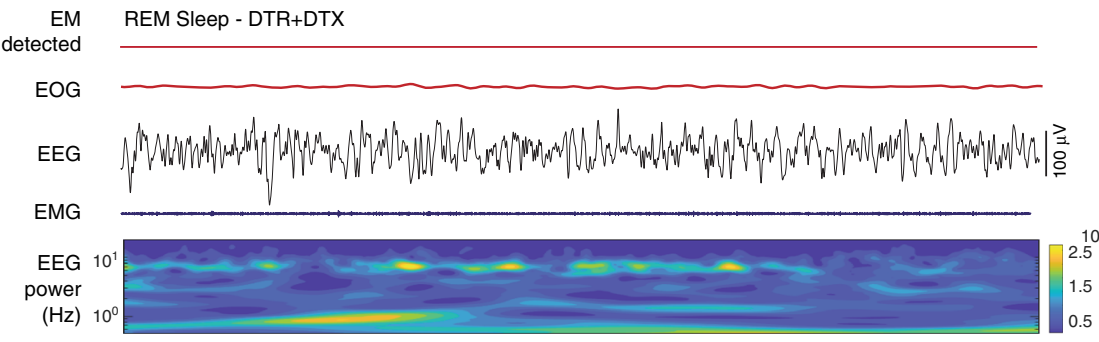

REM Sleep - DTX alone (control)

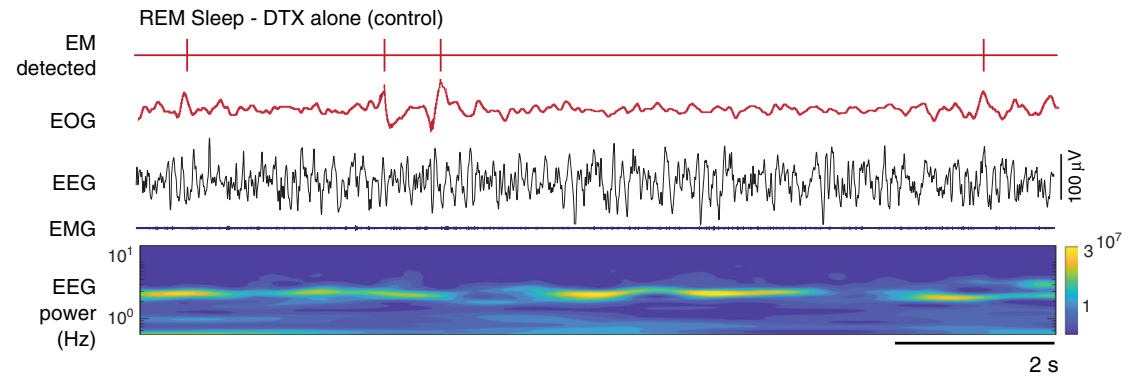

h

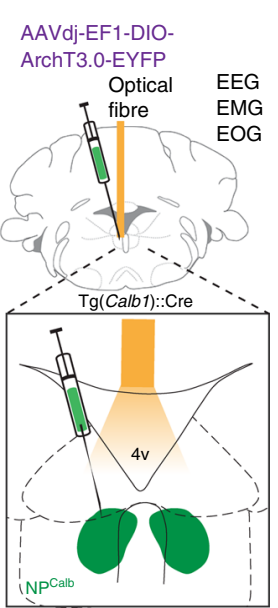

i

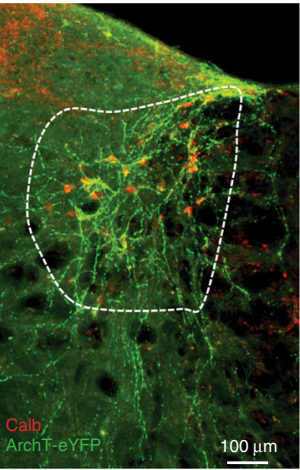

j

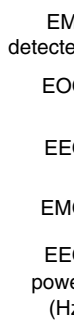

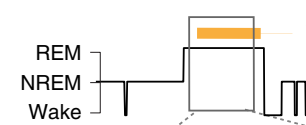

Optical silencing

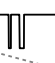

\section{f}
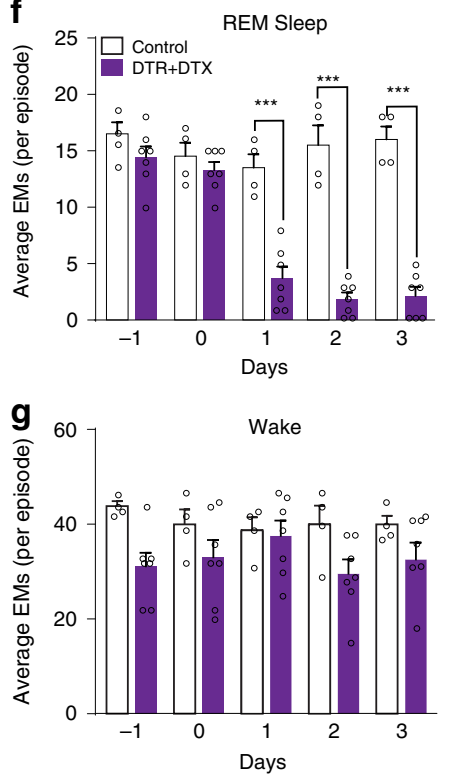

Days
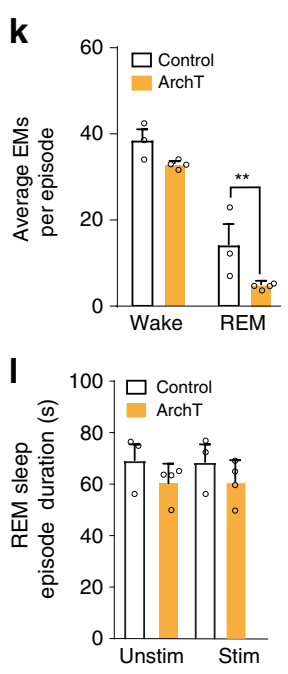

Fig. 5 Genetic ablation and optogenetic silencing of NPCalb neurons block EMs during REM sleep but not wakefulness. a Schematic representation of the genetic targeting of the diphtheria toxin receptor (DTR) expression to NPCalb neurons and implantation of optical fibre. $\mathbf{b}$ Experimental timeline.

c Representative micrographs depicting immunoreactivity for Calb protein in a non-transduced (left) and a DTR-transduced (right) mouse, both i.p.-injected with diphtheria toxin (DTX). Note the remnants of disintegrating neurons in the extracellular space displaying some immunoreactivity for Calb (right). 4V: fourth ventricle; mlf: medial longitudinal fasciculus; NP: Nucleus papilio. $\mathbf{d}$ The number of Calb-expressing neurons is dramatically reduced upon DTX injection in DTR-transduced $(n=3)$ as compared with a wild-type animal that received DTX alone. e Representative experiment showing detected EMs, EOG, EEG and EMG signals, and time frequency heat-map of EEG spectral power from animals transduced with DTR and injected with DTX (upper set of traces) and control mice injected with DTX alone (lower set of traces) during REM sleep. $\mathbf{f}, \mathbf{g}$ Averaged number of EMs during REM sleep $\mathbf{f}$ and wakefulness $\mathbf{g}$, before and after DTX injection ( $n=7$ DTR+/DTX+ and four control animals; Wakefulness: $P=0.523, F(4,36)=0.8171 ; R E M$ sleep: $P \leq 0.001, f(4,36)=19.2)$. ${ }^{\star \star \star} P<0.0001$, two-way ANOVA followed by Sidak's post hoc tests). Genetic ablation of NPCalb neurons does not significantly decrease the number of EMs during wakefulness but eradicate EMs during REM sleep. $\mathbf{h}$ Schematic representation of the genetic targeting of AAV2-Ef1a-DIO-ArchT3.0-EYFP inhibitory opsin expression to NPCalb neurons and implantation of optical fibre. i Representative micrograph showing ArchT-YFP expression (green) in Calbimmunoreactive neurons (red). $\mathbf{j}$ Experimental strategy for optical silencing of NPCalb neurons selectively during REM sleep (top). Detected EMs, EOG, EEG and EMG traces and time frequency heat-map of EEG spectral power (bottom). Orange bar represents the duration of the optical silencing. $\mathbf{k}$ Averaged number of EMs during wakefulness and REM sleep in the control $(n=3)$ and ArchT3.0 $(n=4)$ transduced mice. ${ }^{* \star}, P=0.11$, two-way-ANOVA followed by Sidak's multiple comparison test. Error bars represent SEM values. I Duration of REM sleep episodes under conditions in which pulses of light were either not delivered (unstim.) or delivered (stim.) to silence the NPCalb neurons, in control and transduced mice. Error bars represent SEM values 
$0.02 \%$ sodium-azide, and within which they were maintained until the time of analysis. The sections were immuno-stained according to standard protocols ${ }^{39}$. The following primary antibodies were used in this study: mouse and rabbit antiCalbindin-D28k (CB300 respectively CB38; Dilution: 1:2000; Swant, Marly, Switzerland), mouse and rabbit anti-c-fos (ab208942 respectively PC38, Dilution: 1:2000; respectively, Abcam, Cambridge, UK and Merck, Darmstadt, Germany), rabbit anti-GFP (A11122; Dilution: 1:2000; Life Technologies Europe, Zug, Switzerland), rabbit anti-MCH (H-070-47; Dilution: 1:1000; Phoenix Pharmaceuticals, Karlsruhe, Germany), mouse and rabbit anti-parvalbumin (PV235, respectively, PV28; Dilution: 1:1000; Swant, Marly, Switzerland), mouse anti-tyrosine hydroxylase (22941; Dilution: 1:1000; Immunostar, Houston, USA), rabbit anti-VGlut2 (135403; Dilution: 1:2000; Synaptic Systems, Goettingen, Germany). The secondary antibodies included Cy3-conjugated anti-rabbit and anti-mouse, Cy3- and Cy2conjugated streptavidin, Cy2-conjugated anti-mouse, Alexa488-conjugated antirabbit and anti-mouse (Jackson Immunoresearch, Suffolk, UK), biotinylated antirabbit and anti-mouse (Vector Laboratories, Servion, Switzerland).

The number of Calb-immunoreactive neurons in the brains of various species ( $n=6$ mice; $n=6$ rats; $n=2$ humans) was quantified using Stereoinvestigator 10.52 software (MBF Bioscience, Williston, USA) mounted on a Zeiss photomicroscope, which was equipped with a Hamamatsu Orca OG5 camera.

Deprivation of REM sleep and rebound. Rats (female Wistar rats, Janvier, Lyon, France) and mice (from our animal facility) were deprived of REM sleep by implementing a modified version of the flower-pot technique, which spared the animals of major stress ${ }^{40,41}$. Three groups were established: in the first group ("REM sleep deprivation and rebound" $=$ REMS-D $+\mathrm{R})$, the animals $(n=4$ rats; $n=3$ mice) were maintained together for $72 \mathrm{~h}$ on six small stone platforms $(7 \times$ $4 \mathrm{~cm}$ for rats, $3 \times 3 \mathrm{~cm}$ for mice), placed in a water tank. The surface of the platform was $1 \mathrm{~cm}$ above the water level. During this $72 \mathrm{~h}$ period, the animals had free access to food and water. Owing to the loss of the muscular tone that characterises the onset of REM sleep, the animals fell into the water and were thereby deprived of REM sleep. After $72 \mathrm{~h}$, the animals were transferred to a conventional cage in a quiet room and were permitted for $3 \mathrm{~h}$ to undergo REM sleep (= rebound). In the second group ("REM sleep deprivation" $=$ REMS-D), the animals ( $n=2$ rats; $n=3$ mice) were killed immediately after the termination of the $72 \mathrm{~h}$ REM sleep deprivation period, without recovery. In the third group ("control"), the animals ( $n=3$ rats; $n=3$ mice) were maintained in their cages under standard conditions for $72 \mathrm{~h}$ prior to killed. The animals were anesthetized and perfused with fixative as described above under "Animals" subheading. The brains were then excised and cryosectioned. The sections were immuno-stained for Calb as well as for c-fos, a surrogate marker of neuronal activity ${ }^{42}$. The area within which the cells were counted (on horizontal sections for rats and coronal sections for mouse) was delimited by the boundaries of immunoreactivity for Calb. Within this area the number of cells that manifested immunoreactivity for either Calb alone, c-fos alone, or both Calb and c-fos, were estimated by a single observer in a blinded manner with respect to the treatment conditions. For rats, three alternating horizontal sections through each hemisphere (to avoid counting the same cells on the two sides) were analysed. For mice, the analysis involved 10 alternating coronal sections.

Tract-tracing experiments. Anterograde-tracing experiments were performed on Calb1::Cre mice weighing 25-30 g, following standard protocol ${ }^{39}$. Three successfully injected mice (those in which labelling was confined to the NPCalb neurons) were used for the detailed analysis. One of the two different Cre-dependent viral constructs, namely AAV2/1.CAG.Flex.Tomato.WPRE.bGH or AAV2/1.CAG.Flex. eGFP.WPRE.bGH (Vector Core, University of Pennsylvania, USA), were stereotactically injected in the NPCalb of Calb1::Cre mice at the following Bregma coordinates: rostro-caudal: $-6.36 \mathrm{~mm}$; medio-lateral: $-0.2 \mathrm{~mm}$ and dorso-ventral: $-4.35 \mathrm{~mm}^{14}$. Comparable and reproducible results were obtained with the two viral constructs. Four weeks after the stereotactic injections, the animals were anaesthetised and perfused with paraformaldehyde (PFA) $4 \%$. The brains were excised and cryosectioned and the specimens were analysed by immunofluorescence.

Retrograde tracing was performed using an attenuated rabies virus technique ${ }^{43,44}$. Initially, the NPCalb of Calb1::Cre mice $(n=5)$ was stereotactically injected (at the aforementioned coordinates) with a Cre-dependent helper AAVTVA-Cherry virus. After a lapse of 3 weeks, which sufficed for the expression of the TVA rabies receptor, the following constructs were co-injected: an EnvA- $\Delta \mathrm{G}$ Rabies-GFP virus, a Cre-dependent helper AAV-B19G virus (expressing the B19Gprotein that is necessary for the spreading of the rabies virus to synaptically connected neurons) and (for only two of the five mice) an anterograde tracer (AAV2/1.CAG.Flex.Tomato.WPRE.bGH). Ten days later, the animals were anesthetized and perfused with $4 \%$ PFA. The brains were excised and cryosectioned and the specimens analysed by immunofluorescence.

Optogenetic experimental procedures and in vivo neuronal recording. The Cre-dependent channelrhodopsin viral construct [AAV-EF1a-DIO-hChR2 (H134R)-YFP] was stereotactically injected into the NPCalb of Calb1::Cre mice at the aforementioned coordinates. After 4-6 weeks after injections, animals were chronically implanted with a 200- $\mu$ m fibre implant (MFC-200/245-0.37-5-TF2FLT; Doric Lenses) above the NPCalb (AP: $-6.36 \mathrm{~mm}$, ML: $0 \mathrm{~mm}$; DV: $-4.2 \mathrm{~mm}$ ) or above the $3 \mathrm{~N}$ (AP: $-4.0 \mathrm{~mm}$, ML: $0 \mathrm{~mm}$; DV: $3.0 \mathrm{~mm}$ ), and affixed to the skull with C\&B Metabond (Patterson dental). Then, custom-made EEG/EMG/EOG implants were placed and secured to the skull with dental cement (Patterson dental). EEG signals were recorded from 4 electrodes on the frontal (AP, $-2 \mathrm{~mm}$; $\mathrm{ML}, \pm 2.5 \mathrm{~mm}$ ) and temporal (AP, $3.5 \mathrm{~mm}$; ML $\pm 3 \mathrm{~mm}$ ) cortices. EMG signals were recorded from two electrodes inserted in the neck musculature to record postural tone. EOG signals were recorded from bilaterally implanted stainless steel electrodes with a golden tip wire placed into the orbital cavity to record eye movements. After surgical procedures, mice were allowed to recover in an individual housing cage for at least 2 weeks. After 1 additional week of acclimation to the EEG-EMG recording set up, an optical patch cord (MFP-200/230/900-0.37$2 \mathrm{~m}-\mathrm{FC}-\mathrm{ZF} 1.25$ ) and a zirconia sleeve (ID $=1.25$; Doric Lenses) were connected permanently on the fibre implant. Black nail polish was applied on the plug to blackout the light during the optogenetic stimulation.

To demonstrate the successful optogenetic stimulation of $\mathrm{NP}^{\mathrm{Calb}}$ neurons during in vivo experiments, we injected Calb1::Cre animals with AAV-EF1a-DIOhChR2(H134R)-YFP targeted to the NPCalb (AP: - $6.36 \mathrm{~mm}$, ML: $0 \mathrm{~mm}$; DV: $4.2 \mathrm{~mm}$ ) and then chronically implanted fibre optics and four tetrodes attached to a Microdrive (Nanodrive, Cambridge Neurotech) as well as EEG/EMG four weeks later. Tetrodes were made from four strands of $10-\mu \mathrm{m}$ twisted tungsten wire (CFW0010954, California Fine Wire) connected to an electrode interface board by gold pins (EIB 36, Neuralynx). For data collection mice were connected to a tethered digitising headstage (RHD2132, Intan Technologies) and data sampled at $20 \mathrm{kHz}$ recorded in free open-source software (RHD2000 evaluation software, Intan Technologies). Optical fibres were connected to a patch chord using a zirconia sleeve (Doric Lenses). All recordings were performed in the home cage. The Microdrive was lowered by $50 \mu \mathrm{m}$ and then left to stabilise for $3 \mathrm{~h}$ and then recordings visualised to determine the presence of single-unit neuronal spiking activity. If single-unit neuronal spiking activity was present, a 3-hour baseline sleep-wake period was recorded (sampling rate $20 \mathrm{kHz}$ ). Opto-tagging experiments were performed using blue light $(473 \mathrm{~nm})$ stimulation at $10 \mathrm{~Hz}(10 \mathrm{~ms}$ duration) for $1 \mathrm{~s}$, immediately after baseline recording. Stimulation pulses were delivered at least $10 \mathrm{~s}$ after the onset of each vigilance states (wake, NREM and REM) as determined by online assessment of the EEG/EMG by an experienced experimenter. Stimulation sessions lasted a maximum of $1 \mathrm{~h}$ per day. To determine positive opto-tagging, neuronal spiking activity was first detected and sorted using custom written scripts and activity aligned to laser pulses as recorded by TTL pulses. Neurons that fired within $10 \mathrm{~ms}$ of laser pulses with fidelity of $>80 \%$ were considered to be opto-tagged. The firing characteristics of these opto-tagged neurons across sleep-wake states were then determined from the preceding baseline recording.

Polysomnographic recordings and state transitions. All sleep recordings with optogenetic stimulation took place between 12:00 and 15:00 (light onset at 8:00). EOG, EEG and EMG signals derived from the surgically implanted electrodes were amplified (A-M system) and digitised at $512 \mathrm{~Hz}$ and digitally filtered and spectrally analysed by fast Fourier transformation using custom-made software (View Point Inc.). Polysomnographic recordings were scored using sleep analysis custom-made software (View Point Inc.). All scoring was performed manually based on the visual signature of the EEG and EMG waveforms, as well as the power spectra of 5-s epochs ${ }^{45}$. We defined wakefulness as desynchronized low-amplitude EEG and high tonic EMG activity with phasic bursts. We defined non-REM sleep as synchronised, high-amplitude, low-frequency $(0.5-4 \mathrm{~Hz})$ EEG and highly reduced EMG activity compared with wakefulness with no phasic bursts. We defined REM sleep as having a pronounced theta rhythm $(6-9 \mathrm{~Hz})$ and a flat EMG. State transitions were identified when EEG/EMG criterion changes were predominant for $>50 \%$ of the epoch duration (i.e., 2 s). Polysomnographic scorings were tested by two independent scorers and were found to lie within a $95 \%$ confidence interval ${ }^{45}$. The EOG differential signal deflections were used to quantify EMs. We used Spike 2 software to detect EOG signals in an unsupervised manner. Before peak detection, signals were low filter at $1 \mathrm{~Hz}$ and downsampled to $125 \mathrm{~Hz}$. Smoothing of the signals and a threshold to detect the peak of the eye deflection were applied. The threshold of the deflections was determined based on the mean amplitude of the total of recording time and was adapted per animal. Low-amplitude deflections were not considered for EM quantification. To determine the response times of EM following optical activation of neurons in the NPCalb or its terminals in the $3 \mathrm{~N}$, we determined the times of peak EOG deflection $(>200 \mu \mathrm{V})$ from the onset of the blue light $(473 \mathrm{~nm})$ activation to the first next EM. All detected EMs per animal were aligned to the onset of first light pulse per frequency of stimulation during sleep and wakefulness. Overlay of the average traces per animal of all animals are shown in Figs 3 and 4 and Supplementary Fig. 5. Data collected from all animals were then fitted with a Gaussian curve according to the formula:

$$
y=\text { bin.count } \times \exp \left(\frac{(x-\text { mean } / \text { s.d. })^{2}}{2}\right)
$$

Median curve values were determined from the apex (maximum $y$ value). All plotting and analysis was performed in Graphpad Prism v.6.0. 
Light stimulation procedures. In vivo optogenetic activation of the $\mathrm{NP}^{\mathrm{Calb}}$ was conducted in triplicates. Optical stimulation was delivered using a master-9 pulse generator to deliver $3 \mathrm{mV}$ blue light $(473 \mathrm{~nm})$ using $5 \mathrm{~ms}$ square pulses at $1 \mathrm{~Hz}$, $10 \mathrm{~Hz}$ for $10 \mathrm{~s}$, or $1 \mathrm{~s}$ continuous light-on every minute for 1 hour ${ }^{45}$. Note that animals with aberrant circadian distribution of sleep-wake cycle $(<10 \%)$ were discarded from this study and that these genetic and viral manipulations did not disrupt spontaneous sleep-wake architecture compared to naive and wild-type animals. Animals with the optic fibre above the $3 \mathrm{~N}$ terminals were activated in a state specific manner, where $1 \mathrm{~s}$ continuous light was delivered at the onset of the REM sleep or wake episodes. For the probability, latency and Gaussian distributions analysis, animals with less than two REM sleep episodes in the hour of optogenetic stimulation or with poor EOG signals were not included in the analysis.

The animals for the DTX experiment were injected (AAV9-pCAGFloxedDTRreverse-WPRE) in the NPCalb as described above. Control animals were wild-type C57/Bl6 mice, non-injected but chronically implanted with EOG/EEG and EMG electrodes. After 2 weeks of implantation animals were habituated to the housing cages and recording cables. After 1 week of habituation, we injected $0.1 \mathrm{ml}$ of saline solution i.p. for 3 consecutive days. On the fourth day we injected DTX $(50 \mu \mathrm{g} / \mathrm{kg}$ of body weight) at 11:00 am. Quantification of sleep and EMs were performed on the subsequent hour after injection at the same circadian time. At day 4 post injection, animals were sacrificed for histological verification of the effect of the DTX on DTR-transduced NPCalb neurons.

Inhibition experiments with the silencing construct AAV-EF1a-DIOeArch3.0-EYFP, were done state specifically where the light was delivered at the onset and throughout the wake or the REM sleep episodes and turned off at the time of transition to a different state. Off-line quantification of EMs was calculated by taking the total number of detected EMs during the light-on periods.

Statistical analysis. All behavioural experiments were conducted in at least two cohorts of animals. Statistical significance of comparisons was determined by paired $t$ test (for the experiment consisting of stimulating $\mathrm{NP}^{\text {Calb }}$ terminals in the $3 \mathrm{~N}$ ), and two-way ANOVA (for NPCalb neuron activation, silencing and DTRDTX experiment; $\alpha$ adjusted for multiple comparisons), followed by Sidak multiple comparison post hoc test; $P$ values $0.12 \mathrm{~ns}, 0.33\left(^{*}\right), 0.002\left(^{* *}\right),<0.001\left(^{* * *}\right)$ were considered for significance using 0.001 confidence interval. Exact $P, t$ and $d f$ values are reported in the figure legends.

Image analysis. The specimens were evaluated either in a Leica epifluorescence microscope, a Nikon Eclipse fluorescence microscope, a Leica TCS SP5 confocal laser microscope or a Hamamatsu Nanozoomer scanner. Post-processing of the images and contrast adjustments therein were performed using the Adobe Photoshop and Nanozoomer slide-processing softwares.

Reporting summary. Further information on research design is available in the Nature Research Reporting Summary linked to this article.

\section{Data availability}

All data generated or analysed during this study are included in this published article (and its supplementary information files).

Received: 5 June 2019; Accepted: 25 October 2019;

Published online: 19 November 2019

\section{References}

1. Jouvet, M. Paradoxical sleep-a study of its nature and mechanisms. Prog. Brain Res. 18, 20-62 (1965).

2. Weber, F. \& Dan, Y. Circuit-based interrogation of sleep control. Nature 538, 51-59 (2016)

3. Saper, C. B., Fuller, P. M., Pedersen, N. P., Lu, J. \& Scammell, T. E. Sleep state switching. Neuron 68, 1023-1042 (2010).

4. Boissard, R. et al. The rat ponto-medullary network responsible for paradoxical sleep onset and maintenance: a combined microinjection and functional neuroanatomical study. Eur. J. Neurosci. 16, 1959-1973 (2002).

5. Marquez-Ruiz, J. \& Escudero, M. Tonic and phasic phenomena underlying eye movements during sleep in the cat. J. Physiol. 586, 3461-3477 (2008).

6. Escudero, M. \& Marquez-Ruiz, J. Tonic inhibition and ponto-geniculooccipital-related activities shape abducens motoneuron discharge during REM sleep. J. Physiol. 586, 3479-3491 (2008).

7. Sanchez-Lopez, A. \& Escudero, M. Tonic and phasic components of eye movements during REM sleep in the rat. Eur. J. Neurosci. 33, 2129-2138 (2011).

8. Vanni-Mercier, G. \& Debilly, G. A key role for the caudoventral pontine tegmentum in the simultaneous generation of eye saccades in bursts and associated ponto-geniculo-occipital waves during paradoxical sleep in the cat. Neuroscience 86, 571-585 (1998).

9. Datta, S., Siwek, D. F., Patterson, E. H. \& Cipolloni, P. B. Localization of pontine PGO wave generation sites and their anatomical projections in the rat. Synapse 30, 409-423 (1998).

10. Vanni-Mercier, G., Pelisson, D., Goffart, L., Sakai, K. \& Jouvet, M. Eye saccade dynamics during paradoxical sleep in the cat. Eur. J. Neurosci. 6, 1298-1306 (1994).

11. Gassel, M. M., Marchiafava, P. L. \& Pompeiano, O. Rubrospinal influences during desynchronized sleep. Nature 209, 1218-1220 (1966).

12. Karlsson, K. A., Gall, A. J., Mohns, E. J., Seelke, A. M. \& Blumberg, M. S. The neural substrates of infant sleep in rats. PLoS Biol. 3, el43 (2005).

13. Celio, M. R. Calbindin D-28k and parvalbumin in the rat nervous system. Neuroscience 35, 375-475 (1990).

14. Watson, C. \& Paxinos, G. Chemoarchitectonic atlas of the mouse brain. (Academic Press - Elsevier, Amsterdam, 2010).

15. Paxinos, G., Watson, C., Carrive, P., Kirkcaldie, M. \& Ashwell, K. W. S. Chemoarchitectonic atlas of the rat brain. 2nd ed (Elsevier - Academic Press, Amsterdam, 2009).

16. Verret, L., Leger, L., Fort, P. \& Luppi, P. H. Cholinergic and noncholinergic brainstem neurons expressing Fos after paradoxical (REM) sleep deprivation and recovery. Eur. J. Neurosci. 21, 2488-2504 (2005).

17. Clement, $\mathrm{O}$. et al. The inhibition of the dorsal paragigantocellular reticular nucleus induces waking and the activation of all adrenergic and noradrenergic neurons: a combined pharmacological and functional neuroanatomical study. PLoS. ONE 9, e96851 (2014).

18. Gervasoni, D. et al. Electrophysiological evidence that noradrenergic neurons of the rat locus coeruleus are tonically inhibited by GABA during sleep. Eur. J. Neurosci. 10, 964-970 (1998).

19. Goutagny, R. et al. Role of the dorsal paragigantocellular reticular nucleus in paradoxical (rapid eye movement) sleep generation: a combined electrophysiological and anatomical study in the rat. Neuroscience 152, 849-857 (2008).

20. Luppi, P. H. et al. The neuronal network responsible for paradoxical sleep and its dysfunctions causing narcolepsy and rapid eye movement (REM) behavior disorder. Sleep Med. Rev. 15, 153-163 (2011).

21. Pedersen, N. P., Anaclet, C., Saper, C. B. \& Lu, J. Circuitry underlying rapid eye movements during REM sleep. Sleep 32, A25 (2009).

22. Hittinger, M. \& Horn, A. K. The anatomical identification of saccadic omnipause neurons in the rat brainstem. Neuroscience 210, 191-199 (2012).

23. Buttner-Ennever, J. A., Horn, A. K., Scherberger, H. \& d'Ascanio, P. Motoneurons of twitch and nontwitch extraocular muscle fibres in the abducens, trochlear, and oculomotor nuclei of monkeys. J. Comp. Neurol. 438, 318-335 (2001).

24. Fraigne, J. J., Torontali, Z. A., Snow, M. B. \& Peever, J. H. REM sleep at its core circuits, neurotransmitters, and pathophysiology. Front. Neurol. 6, 123 (2015).

25. Adamantidis, A. \& de Lecea, L. Sleep and metabolism: shared circuits, new connections. Trends Endocrinol. Metab. 19, 362-370 (2008).

26. Vanni-Mercier, G., Debilly, G., Lin, J. \& Pelisson, D. The caudo-ventral pontine tegmentum is involved in the generation of high velocity eye saccades in bursts during paradoxical sleep in the cat. Neurosci. Lett. 213, 127-131 (1996).

27. Marquez-Ruiz, J. \& Escudero, M. Eye movements and abducens motoneuron behavior after cholinergic activation of the nucleus reticularis pontalis caudalis. Sleep 33, 1517-1527 (2010).

28. Escudero, M., de la Cruz, R. \& Delgado-Garcia, J. A physiological study of vestibular and prepositus hypoglossi neurons projecting to the abducens nucleus in the alert cat. J. Physiol. 458, 539-560 (1992).

29. Webster, H. H. \& Jones, B. E. Neurotoxic lesions of the dorsal pontomesencephalic tegmentum-cholinergic cell area in the cat. II. Effects upon sleep-waking states. Brain Res. 458, 285-302 (1988).

30. Shouse, M. N. \& Siegel, J. M. Pontine regulation of REM sleep components in cats: integrity of the pedunculopontine tegmentum (PPT) is important for phasic events but unnecessary for atonia during REM sleep. Brain Res. 571, 50-63 (1992).

31. Siegel, J. M., Tomaszewski, K. S. \& Nienhuis, R. Behavioral states in the chronic medullary and mid-pontine cat. Electroencephalogr. Clin. Neurophysiol. 63, 274-278 (1986).

32. Suzuki, Y. et al. Deficits in torsional and vertical rapid eye movements and shift of Listing's plane after uni- and bilateral lesions of the rostral interstitial nucleus of the medial longitudinal fasciculus. Exp. Brain Res. 106, 215-232 (1995).

33. Scudder, C. A., Fuchs, A. F. \& Langer, T. P. Characteristics and functional identification of saccadic inhibitory burst neurons in the alert monkey. J. Neurophysiol. 59, 1430-1454 (1988).

34. Strassman, A., Highstein, S. M. \& McCrea, R. A. Anatomy and physiology of saccadic burst neurons in the alert squirrel monkey. I. Excitatory burst neurons. J. Comp. Neurol. 249, 337-357 (1986). 
35. Horn, A. K., Buttner-Ennever, J. A., Suzuki, Y. \& Henn, V. Histological identification of premotor neurons for horizontal saccades in monkey and man by parvalbumin immunostaining. J. Comp. Neurol. 359, 350-363 (1995).

36. Ioannides, A. et al. MEG tomography of human cortex and brainstem activity in waking and REM sleep saccades. Cereb. Cortex 14, 56-72 (2004).

37. Hong, C. C. H. et al. fMRI evidence for multisensory recruitment associated with rapid eye movements during sleep. Hum. Brain Mapp. 30, 1705-1722 (2009).

38. Datta, S. \& Hobson, J. A. Neuronal activity in the caudo-lateral peribrachial pons: relationship to PGO waves and rapid eye movement. J. Neurophysiol. 71, 95.109 (1994).

39. Bilella, A., Alvarez-Bolado, G. \& Celio, M. R. The Foxb1-expressing neurons of the ventrolateral hypothalamic parvafox nucleus project to defensive circuits. J. Comp. Neurol. 524, 2955-2981 (2016).

40. Mendelson, W. B., Guthrie, R. D., Frederick, G. \& Wyatt, R. J. The flower pot technique of rapid eye movement (REM) sleep deprivation. Pharmacol. Biochem. Behav. 2, 553-556 (1974).

41. Arthaud, S. et al. Paradoxical (REM) sleep deprivation in mice using the small-platforms-over-water method: polysomnographic analyses and melanin-concentrating hormone and hypocretin/orexin neuronal activation before, during and after deprivation. J. Sleep. Res. 24, 309-319 (2015).

42. Maloney, K. J., Mainville, L. \& Jones, B. E. c-fos expression in dopaminergic and GABAergic neurons of the ventral mesencephalic tegmentum after paradoxical sleep deprivation and recovery. Eur. J. Neurosci. 15, 774-778 (2002).

43. Wall, N. R., Wickersham, I. R., Cetin, A., De La Parra, M. \& Callaway, E. M. Monosynaptic circuit tracing in vivo through Cre-dependent targeting and complementation of modified rabies virus. Proc. Natl. Acad. Sci. USA 107, 21848-21853 (2010).

44. Wickersham, I. R., Finke, S., Conzelmann, K. K. \& Callaway, E. M. Retrograde neuronal tracing with a deletion-mutant rabies virus. Nat. Methods 4, 47-49 (2007).

45. Herrera, C. G., Ponomarenko, A., Korotkova, T., Burdakov, D. \& Adamantidis, A. Sleep \& metabolism: the multitasking ability of lateral hypothalamic inhibitory circuitries. Front. Neuroendocrinol. 44, 27-34 (2016).

\section{Acknowledgements}

We thank Professor Claudio Bassetti (Bern), for his continuous interest in this project and for promoting and supporting the collaboration between our two research groups. The technical, scientific, and administrative assistance of Simone Eichenberger, Laurence Clément, Christiane Marti, Rachel Ververidis, Ludiwine Aeby, Séverin Rossi, Samara Naim, Michelle Kueffer, Dr. Rosario Arévalo, and Dr. Andrina Zbinden are gratefully acknowledged. Dr A. Babalian performed most of the stereological injections. Dr. S. Arber, (FMI, Basel) kindly supplied several viral constructs, e.g., the DTR. Drs. Andreas Lüthi and Chung Xu (FMI, Basel) furnished us with information and reagents to conduct the experiments with the Rabies virus. This project was funded by the Canton of Fribourg
(Switzerland), the Swiss National Foundation (31003A-144036 and 320030-179565 to M.R.C. and 31003-156156 to A.A.), the University of Bern (IRC to C.G.H and A.A.), the Inselspital (to A.A.) and a Consolidator Grant from the European Research Council (to A.A.).

\section{Author contributions}

Conceptualisation: F.G, C.G.H, M.R.C; investigation: F.G, C.G.H, A.B., T.G., D.R.W, M.R.C; resources: A.A, M.R.C; writing-original draft: F.G, C.G.H, A.A., M.R.C; writingreview and editing: F.G, C.G.H, A.A, M.R.C; visualisation: F.G, C.G.H; supervision: F.G, M.R.C; veterinary authorisation: M.R.C, A.A.; funding acquisition: M.R.C, A.A.

\section{Competing interests}

The authors declare no competing interests.

\section{Additional information}

Supplementary information is available for this paper at https://doi.org/10.1038/s41467019-13217-y.

Correspondence and requests for materials should be addressed to F.G. or M.R.C.

Peer review information Nature Communications thanks Michael Lazarus, Pierre-Hervé Luppi and Jerome Siegel for their contribution to the peer review of this work. Peer reviewer reports are available.

Reprints and permission information is available at http://www.nature.com/reprints

Publisher's note Springer Nature remains neutral with regard to jurisdictional claims in published maps and institutional affiliations.

(c) (i) Open Access This article is licensed under a Creative Commons Attribution 4.0 International License, which permits use, sharing, adaptation, distribution and reproduction in any medium or format, as long as you give appropriate credit to the original author(s) and the source, provide a link to the Creative Commons license, and indicate if changes were made. The images or other third party material in this article are included in the article's Creative Commons license, unless indicated otherwise in a credit line to the material. If material is not included in the article's Creative Commons license and your intended use is not permitted by statutory regulation or exceeds the permitted use, you will need to obtain permission directly from the copyright holder. To view a copy of this license, visit http://creativecommons.org/ licenses/by/4.0/.

(C) The Author(s) 2019 